\section{Original Article}

Check for updates

\section{OPEN ACCESS}

Received: Jun 3, 2018

Revised: Aug 10, 2018

Accepted: Aug 18, 2018

*Correspondence to

Jun-Young Seo

Severance Biomedical Science Institute, Brain Korea 21 PLUS Project for Medical Science, Yonsei University College of Medicine, 50-1 Yonsei-ro, Seodaemun-gu, Seoul 03722, Korea. E-mail: jyseo0724@yuhs.ac

†John Eom and Jihye Yoo contributed equally to this work.

Copyright (c) 2018. The Korean Association of Immunologists

This is an Open Access article distributed under the terms of the Creative Commons Attribution Non-Commercial License (https:// creativecommons.org/licenses/by-nc/4.0/) which permits unrestricted non-commercial use, distribution, and reproduction in any medium, provided the original work is properly cited.

Conflict of Interest

The authors declare no potential conflicts of interest.

\section{Abbreviations}

Arg-1, arginase-1; BMDM, bone marrowderived macrophages; FBS, fetal bovine serum; GM-CSF, granulocyte macrophage colony-stimulating factor; IFN, interferon; iNOS, inducible nitric oxide synthase; IRF, interferon regulatory factor; $\mathrm{KO}$, knockout; LPS, lipopolysaccharides; M-CSF, macrophage colony-stimulating factor; MHC, major histocompatibility complex; SEM, standard error of the mean; TGF,

\section{Viperin Deficiency Promotes Polarization of Macrophages and Secretion of M1 and M2 Cytokines}

\author{
John Eom ${ }^{+}$, Jihye Yoo $^{+}$, Jeong Jin Kim, Jae Bong Lee, Wanho Choi, Chae Gyu Park, \\ Jun-Young Seo*
}

Severance Biomedical Science Institute, Brain Korea 21 PLUS Project for Medical Science, Yonsei University College of Medicine, Seoul 03722, Korea

\section{ABSTRACT}

Viperin is a multifunctional protein that was first identified in human primary macrophages treated with interferon- $\gamma$ and in human fibroblasts infected with human cytomegalovirus. This protein plays a role as an anti-viral protein and a regulator of cell signaling pathways or cellular metabolism when induced in a variety of cells such as fibroblasts, hepatocytes and immune cells including $\mathrm{T}$ cells and dendritic cells. However, the role of viperin in macrophages is unknown. Here, we show that viperin is basally expressed in murine bone marrow cells including monocytes. Its expression is maintained in bone marrow monocyte-derived macrophages (BMDMs) depending on macrophage colony-stimulating factor (M-CSF) treatment but not on granulocyte-macrophage colony-stimulating factor (GM-CSF) treatment. In wild type (WT) and viperin knockout (KO) BMDMs differentiated with M-CSF or G-MCSF, there are little differences at the gene expression levels of M1 and M2 macrophage markers such as inducible nitric oxide synthase (iNOS) and arginase-1, and cytokines such as IL-6 and IL-10, indicating that viperin expression in BMDMs does not affect the basal gene expression of macrophage markers and cytokines. However, when BMDMs are completely polarized, the levels of expression of macrophage markers and secretion of cytokines in viperin KO M1 and M2 macrophages are significantly higher than those in WT M1 and M2 macrophages. The data suggest that viperin plays a role as a regulator in polarization of macrophages and secretion of M1 and M2 cytokines.

Keywords: Viperin; Macrophage; iNOS; Arginase-1; IL-6; IL-10

\section{INTRODUCTION}

Macrophages play a critical role in antigen presentation, phagocytosis, and immunomodulation (1). They can be polarized into classically activated macrophages (M1) or alternatively activated macrophages (M2) in response to different stimulation (2). M1 and M2 macrophages have different roles in inflammation. M1 macrophages are associated with production of pro-inflammatory cytokines such as tumor necrosis factor (TNF)- $\alpha$, IL-6, and IL-12 and provide a host defense against infections, while M2 macrophages secrete anti-inflammatory cytokines such as transforming growth factor (TGF)- $\beta$ and IL-10 and have reparative function $(3,4)$. M1 macrophages stimulated by interferon (IFN)- $\gamma$ and/or 
transforming growth factor; TNF, tumor

necrosis factor; WT, wild type

Author Contributions

Conceptualization: Eom J, Seo JY; Data curation: Eom J, Yoo J, Seo JY; Formal analysis: Eom J, Yoo J; Funding acquisition: Seo JY; Investigation: Eom J, Yoo J, Kim JJ, Lee JB, Choi W, Park CG, Seo JY; Supervision: Seo JY; Validation: Eom J, Yoo J; Writing - original draft: Yoo J; Writing - review \& editing: Seo JY. lipopolysaccharides (LPS) or microbial product express high levels of CD68 marker, CD80 and CD86 costimulatory molecules, major histocompatibility complex (MHC) class II, and inducible nitric oxide synthase (iNOS) $(3,4)$. M2 macrophages induced by IL-4 or IL-13 express increased levels of arginase-1 (Arg-1), CD206 mannose receptor, and CD163 marker $(3,4)$. Moreover, recent studies showed that M1 and M2 macrophages can be converted into each other in their specific microenvironment (5-7).

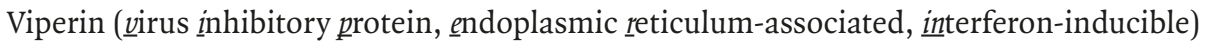
was first identified as a gene induced by IFN- $\gamma$ in human primary macrophages and by human cytomegalovirus infection in human fibroblasts $(8,9)$. It is also induced in a variety of cells by type I, II, and III IFNs, double-stranded (ds) B-form DNA, the dsRNA analog polyinosinicpolycytidylic acid, LPS, and many other viral infections (10). Viperin is a multifunctional protein. It functions as an anti-viral protein against many viruses in the majority of cell types including fibroblasts, hepatocytes, and astrocytes $(8,11-14)$. This protein also plays a role as a regulator of cell signaling pathways in plasmacytoid dendritic cells and $\mathrm{T}$ helper 2 cells $(15,16)$. Moreover, it functions as a pro-viral protein by modulating cellular energy and lipid metabolism in fibroblasts infected with human cytomegalovirus $(17,18)$. Although viperin's functions in a variety of cell types were well known, the role of viperin in macrophages in which it was identified remains to be elucidated.

In this study, we showed that viperin is expressed in murine bone marrow cells including monocytes, and its expression is kept in bone marrow-derived macrophages (BMDMs) differentiated with macrophage colony-stimulating factor (M-CSF). Using BMDMs prepared from viperin knockout $(\mathrm{KO})$ mice, we found that viperin deficiency facilitates polarization of BMDMs into M1 and M2 macrophages and secretion of cytokines, suggesting that viperin has a pivotal role as a regulator in macrophage polarization.

\section{MATERIALS AND METHODS}

\section{Cells, antibodies, and reagents}

Murine bone marrow cells used in the study were isolated from wild type (WT) or viperin (also known as Rsad2) KO C57BL/6 mice (18).

Viperin was detected with the monoclonal mouse antibody to viperin (MaP.VIP) described previously $(8,13)$. The monoclonal mouse anti- $\beta$-actin antibody (Sigma-Aldrich, St. Louis, MO, USA), horseradish peroxidase-conjugated goat anti-rabbit or anti-mouse IgG antibody (Jackson ImmunoResearch Laboratories, West Grove, PA, USA), PE-conjugated anti-Ly-6C or anti-F4/80 antibody (BioLegend, San Diego, CA, USA), and PerCP-Cy5.5-conjugated antiCD11b antibody (BD Biosciences, Franklin Lakes, NJ, USA) were used.

Mouse M-CSF or granulocyte macrophage colony-stimulating factor (GM-CSF) is stably expressed in CHO cells as described previously (19). The culture supernatants containing the secreted M-CSF or GM-CSF were used for generation of BMDMs.

\section{Polarization of M1 and M2 macrophages}

To generate BMDMs, the bone marrow cells from femurs and tibias of mice were harvested and cultured in RPMI (Hyclone ${ }^{\mathrm{TM}}$; GE Healthcare, Chicago, IL, USA) supplemented with $10 \%$ fetal bovine serum (FBS) (Hyclone ${ }^{\mathrm{TM}}$; GE Healthcare), 1\% penicillin/streptomycin 
(Sigma-Aldrich), and 10\% M-CSF or GM-CSF supernatants for 7 days. BMDMs cultured in M-CSF conditioned media were washed and plated at a density of 5-6 $\times 10^{6}$ cells/well in 6-well plate (SPL Life Sciences, Pocheon, Korea) containing RPMI media without M-CSF. For M1 polarization, cells were classically activated with $100 \mathrm{ng} / \mathrm{ml} \mathrm{LPS} \mathrm{(Sigma-Aldrich)} \mathrm{+} 20 \mathrm{ng}$ / ml IFN- $\gamma$ (R\&D Systems, Minneapolis, MN, USA) for $24 \mathrm{~h}$. For M2 polarization, cells were alternatively activated with $20 \mathrm{ng} / \mathrm{ml}$ IL-4 (PrimeGene, Shanghai, China) for $48 \mathrm{~h}$.

\section{Flow cytometry}

BMDMs, M1 or M2 macrophages were blocked with 2.4G2 blocking buffer for 15 min at $4^{\circ} \mathrm{C}$. Then, cells were surface stained with fluorescently conjugated FACS antibodies including V450-conjugated anti-CD86, FITC-conjugated anti-MHC II, and FITC-conjugated antiCD206 antibodies and isotype control antibodies for $30 \mathrm{~min}$ at $4^{\circ} \mathrm{C}$ in dark condition. Cells were washed with $150 \mathrm{ul} \mathrm{FACS}$ buffer (1\% FBS in PBS) and centrifuged at 2,000 rpm for 2 min. After washing with FACS buffer, single cell suspensions were obtained with cell strainers and resuspended in FACS buffer. Then cells were run on a LSR-II Fortessa Flow Cytometer (BD Biosciences). Data were analyzed with FlowJo software.

\section{Cytometric bead array}

To measure the levels of M1 and M2 secrete cytokines, the mouse TNF- $\alpha$, IL- 6 , and IL10 flex kits (BD Biosciences) were used. The assays were performed according to the manufacturer's instructions. Samples and standards were processed on the BD FACSVerse Flow Cytometer (BD Biosciences). Data were analyzed with FlowJo software (FlowJo, LLC, Ashland, OR, USA).

\section{Immunoblot analysis}

Cells were harvested and lysed in lysis buffer ( $1 \%$ Triton X-100 in TBS). The concentrations of protein were measured by bicinchoninic acid assay (Thermo Fisher Scientific, Waltham, MA, USA). The proteins were separated by $10 \%$ SDS-polyacrylamide gel electrophoresis gels and transferred to polyvinylidene difluoride membranes. The blots were blocked in $5 \%$ skim milk and $0.05 \%$ Tween in PBS for $1 \mathrm{~h}$, incubated with primary antibodies, and probed with horseradish peroxidase-conjugated secondary antibodies, followed by incubation with enhanced chemiluminescence reagents (Thermo Fisher Scientific).

\section{RNA extraction, CDNA preparation, and quantitative real-time PCR}

Cells were harvested and total RNA were isolated using RNeasy Mini kit (Qiagen, Hilden, Germany). The cDNA synthesis was performed with 1 ug RNA using Prime script 1st strand cDNA synthesis kit according to the manufacturer's instructions (Takara Bio Inc., Kusatsu, Japan). The cDNA obtained from cells was quantified by quantitative real-time PCR using SYBR Green PCR kit (Applied Biosystems, Foster City, CA, USA). The reaction included $95^{\circ} \mathrm{C}$ for $10 \mathrm{~min}$, which was followed by a 3-step PCR program of $95^{\circ} \mathrm{C}$ for $30 \mathrm{~s}, 55^{\circ} \mathrm{C}$ for 1 $\mathrm{min}$, and $72^{\circ} \mathrm{C}$ for $30 \mathrm{~s}$ repeated for 50 cycles. The PCR was performed in triplicate for each sample. Quantitation was performed by the comparative $\mathrm{Ct}\left(2^{-\Delta \Delta C t}\right)$ method. The Ct value for each sample was normalized by the value for $\beta$ actin gene. Two independent experiments were analyzed statistically for differences in the mean values, and $\mathrm{p}$ values are indicated in the figures.

\section{Statistical analysis}

The data are presented as mean \pm standard error of the mean (SEM). Statistical significance was determined using unpaired 2-tailed Student's $t$-test. $\mathrm{p}<0.05$ was considered significant. 


\section{RESULTS}

\section{Viperin is basally expressed in murine bone marrow cells and BMDMS.}

Viperin was identified in human primary macrophages treated with IFN- $\gamma(8)$. To investigate the role of viperin in the macrophages, we isolated murine bone marrow cells from mice and assessed viperin expression in each cell population. Viperin expression was detected in most bone marrow cells including granulocytes (CD11 b $\left.{ }^{\text {high }} \mathrm{Ly} 6 \mathrm{C}^{\text {middle }}, 19.1 \%\right)$, monocytes (CD11b ${ }^{\text {middle }} \mathrm{Ly6C} \mathrm{C}^{\text {high }}, 4.51 \%$ ), and other cell populations (CD11b ${ }^{\text {low }} \mathrm{Ly} 6 \mathrm{C}^{\text {low }}$; CD11 $\mathrm{b}^{\text {high }} \mathrm{Ly} 6 \mathrm{C}^{\text {low }}$; CD11 $\left.{ }^{\text {low }}{ }^{2} 6 \mathrm{C}^{\text {high }}\right)$ in the absence of IFN treatment (Fig. 1A). It has been well established that bone marrow cells differentiate into macrophages in M-CSF or GM-CSF conditioned media (20-22). Viperin expression was maintained in the BMDMs $\left(\mathrm{F} 4 / 80^{+} \mathrm{CD} 11 \mathrm{~b}^{+}\right)$differentiated

A
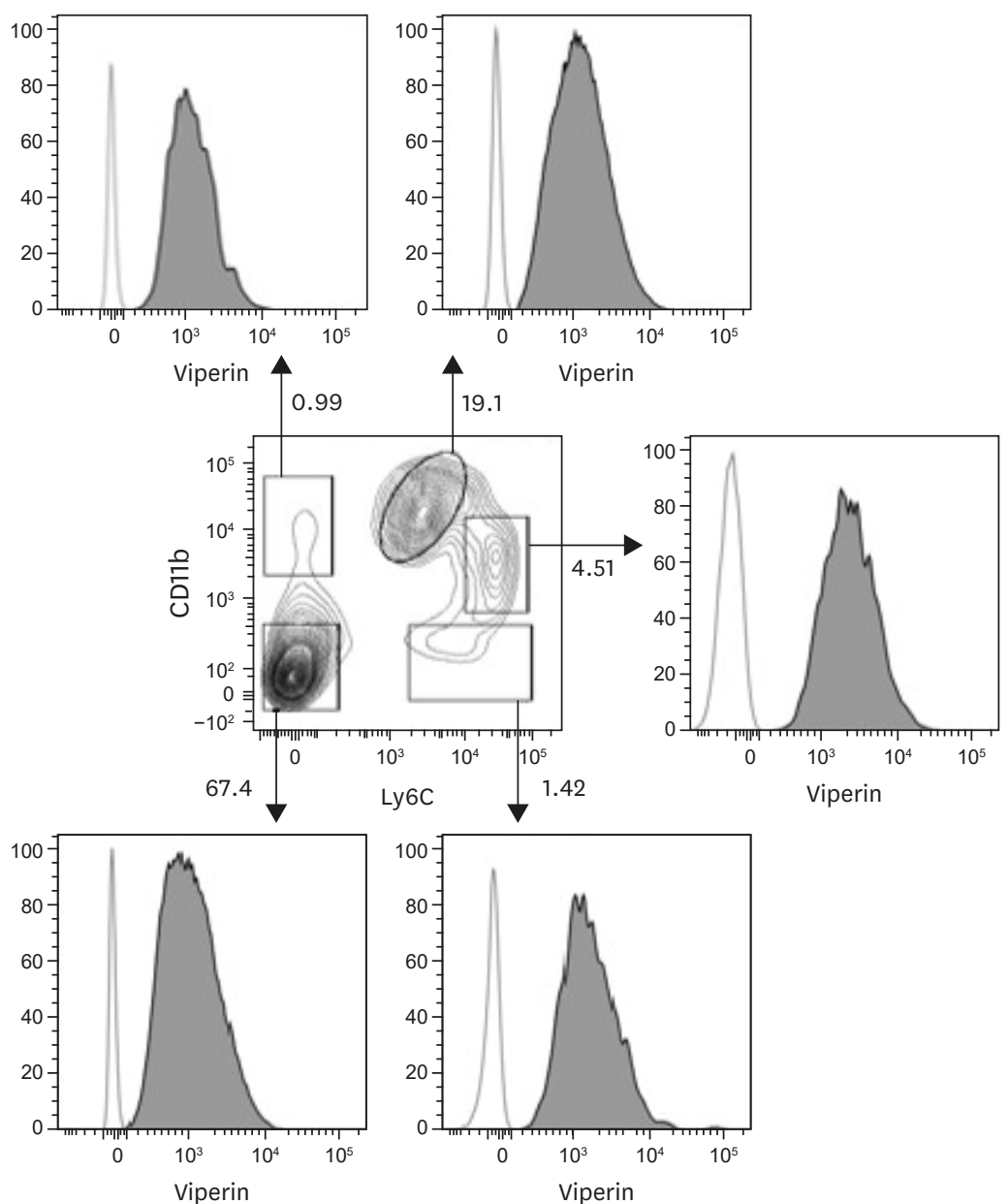

B

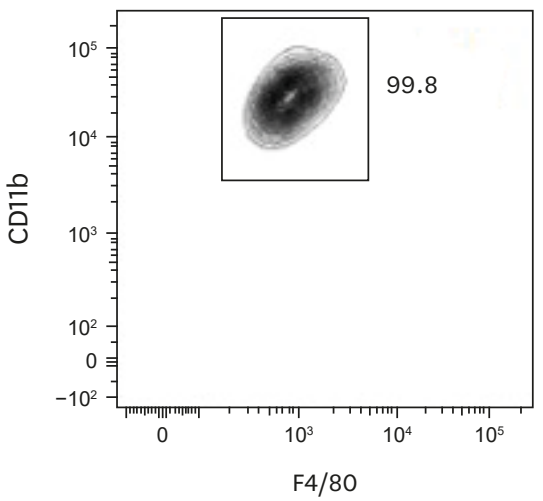

C

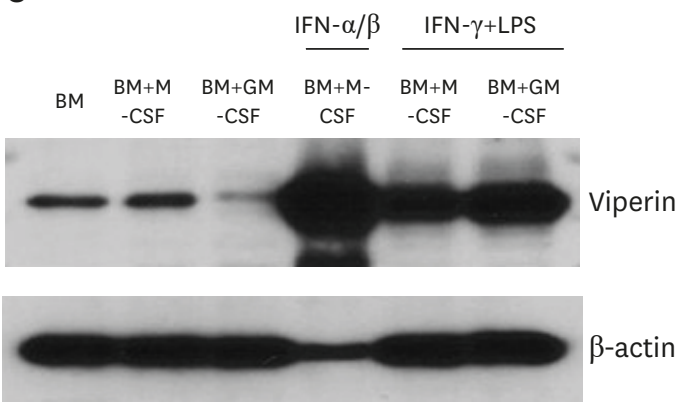

Figure 1. Viperin expression in murine bone marrow cells and BMDMs. (A) The expression of viperin in bone marrow cells. The murine bone marrow cells were isolated from WT C57BL/6 mice. The cells were stained with fluorescently-conjugated antibodies specific to CD11b, Ly6C, and viperin and analyzed by flow cytometry. Cell fractions were gated in a flow cytogram. Specific cell types were confirmed by expression levels of CD11b and Ly6C (Granulocytes, CD11b ${ }^{\text {high }}$ Ly6Cmiddle; Monocytes, $\mathrm{CD}_{11} \mathrm{~b}^{\text {middle }}$ Ly $\left.6 \mathrm{C}^{\text {high }}\right)$. Viperin expression of each cell population was shown in flow histograms. Shaded histograms represent staining with anti-viperin antibody; open histograms represent staining with an isotype-matched control antibody of irrelevant specificity. Data are representative of two independent experiments. (B, C) The expression of viperin in BMDMs. The bone marrow cells were differentiated into BMDMs by incubation in M-CSF or GM-CSF conditioned media for 7 days. The cells were stained with fluorescently-conjugated antibodies specific to CD11b and F4/80. Cell fractions were analyzed by flow cytometry. BMDMs specificity was confirmed by expression of F4/80 and CD11b (F4/80 $\left.\mathrm{CD}_{11 \mathrm{~b}}\right)$. BMDMs were washed and plated in 6-well plates containing media without M-CSF or GM-CSF. The cells were treated with or without type I IFN $(1,000 \mathrm{U} / \mathrm{ml})$ for $8 \mathrm{~h}$ or IFN- $\gamma(20 \mathrm{ng} / \mathrm{ml})$ and LPS $(100 \mathrm{ng} / \mathrm{ml})$ for $24 \mathrm{~h}$. Viperin expression in BMDMs was detected by immunoblot using monoclonal antibody specific to viperin (MaP.VIP). $\beta$-actin served as a protein-loading control.

$\mathrm{BM}$, bone marrow cells; BM+M-CSF, BMDMs differentiated with M-CSF; BM+GM-CSF, BMDMs differentiated with GM-CSF under the indicated conditions. 
with M-CSF but not with GM-CSF (Fig. 1B and C). Consistent with previous reports $(8,13)$, viperin expression was significantly increased in BMDMs differentiated with M-CSF or GMCSF in the presence of IFN and/or LPS treatment (Fig. 1C). The results indicated that viperin is basally expressed in murine bone marrow cells and its expression is maintained in BMDMs differentiated with M-CSF.

\section{Viperin has little effect on the phenotypes of BMDMs.}

To examine the effects of viperin on expression of macrophage markers and inflammatory cytokines in BMDMs, we isolated bone marrow cells from WT and viperin KO mice and differentiated into BMDMs in GM-CSF or M-CSF conditioned media. There were little differences in the expression levels of $i N O S, C d 80$, and $C d 86$ known as M1 macrophage markers, Tnf-a, Il-1b, Il-6, Il-12, and Il-23 known as M1 macrophage cytokines, Cd163, Cd206, and $\mathrm{Arg}-1$ known as M2 macrophage markers, and $I l-10$ and Tgf- $b$ known as M2 macrophage cytokines between WT and viperin KO BMDMs differentiated with GM-CSF (Fig. 2A). Similar results were obtained from WT and viperin KO BMDMs differentiated with M-CSF, although the expression levels of Tnf- $a$ and $I l-23$ cytokines in viperin KO BMDMs had a twofold decrease (Fig. 2B). The results indicated that viperin expression is not required for the phenotypes of BMDMs differentiated with GM-CSF or M-CSF.

A

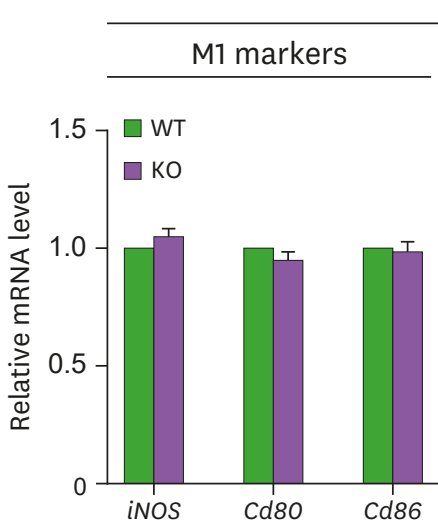

GM-CSF

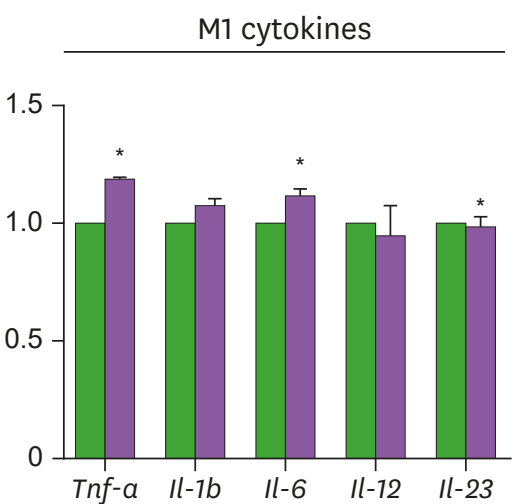

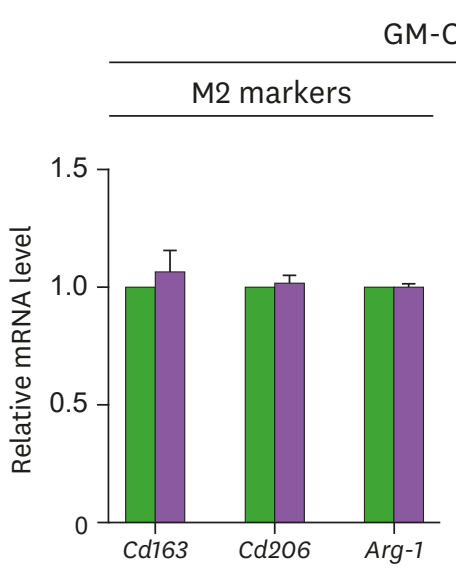

GM-CSF

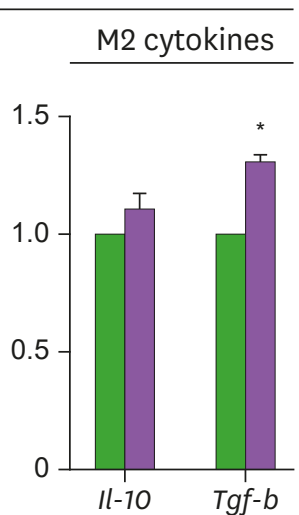

B

M-CSF

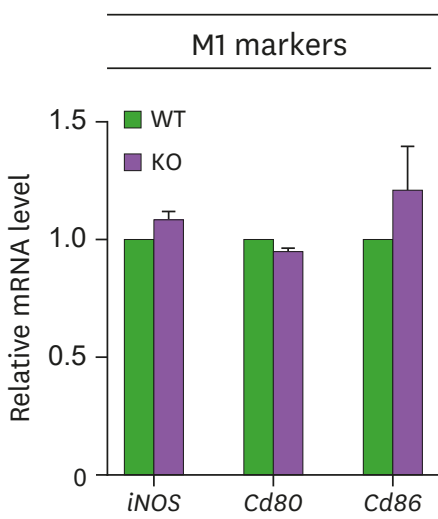

M1 cytokines

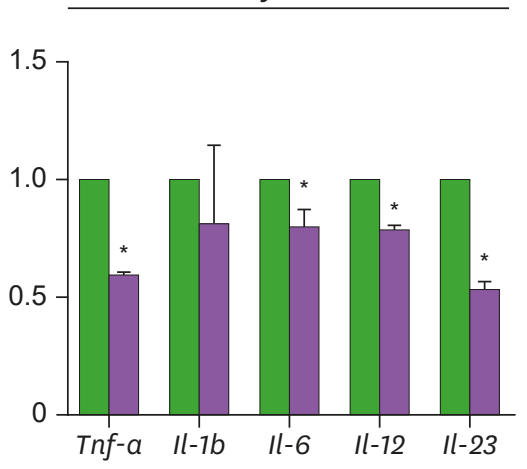

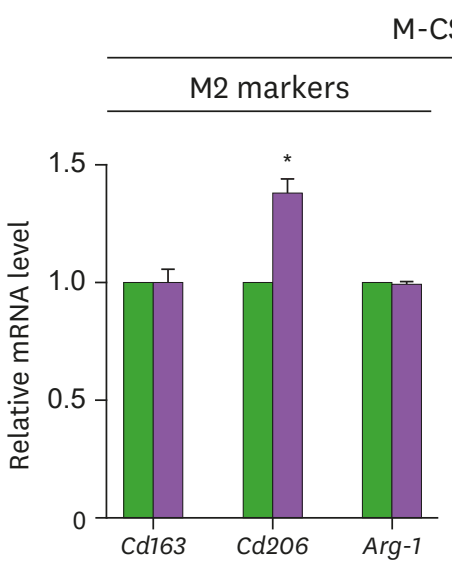

M-CSF

M2 cytokines

Figure 2. Viperin effects on the BMDMs differentiated with M-CSF or GM-CSF. The bone marrow cells were isolated from WT and viperin KO C57BL/6 mice and differentiated into BMDMs in conditioned media. (A, B) The effects of viperin on expression of macrophage markers and cytokines. The mRNA expression levels of M1 macrophage markers and cytokines (left) and M2 macrophage markers and cytokines (right) in BMDMs differentiated with GM-CSF (A) or M-CSF (B) were measured by quantitative RT-PCR and normalized to $\beta$-actin mRNA. Data are presented as mean \pm SEM of triplicate samples and are representative of two individual experiments. ${ }^{*} \mathrm{p}<0.05$. 
Viperin deficiency promotes gene expression of macrophage markers and cytokines in the polarized M1 and M2 macrophages.

It is well known that IFN- $\gamma$, LPS, and IL-4 stimulation can differentially induce complete M1 and M2 polarization (23). To examine viperin effects in completely polarized M1 and M2 macrophages, we stimulated WT and viperin KO BMDMs with IFN- $\gamma$ and LPS for $24 \mathrm{~h}$ and with IL-4 for $48 \mathrm{~h}$. Viperin expression was highly increased in M1 macrophages, indicating that viperin, an IFN-inducible protein is expressed by Toll-like receptor and IFN signaling pathways under IFN- $\gamma$ and LPS treatments. However, viperin expression was reduced in M2 macrophages, suggesting that its expression is unable to be maintained by IL-4 in the absence of M-CSF (Fig. 3A). Polarization of BMDMs into M1 and M2 macrophages was assessed by measuring the expression levels of M1 and M2 markers (Fig. 3B). As expected, iNOS, an M1 marker was upregulated in both WT and viperin KO M1 macrophages but not in M2 macrophages. Arg-1, an M2 marker was upregulated in both WT and viperin KO M2 macrophages but not in M1 macrophages as well. Interestingly, the expression levels of iNOS and $\mathrm{Arg}-1$ in viperin KO M1 and M2 macrophages were highly increased, when compared to those in WT macrophages (Fig. 3B). To further analyze this phenotype, we examined gene expression of macrophage markers and cytokines in WT and viperin KO M1 and M2 macrophages (Fig. 3C). The expression levels of M1 macrophage markers, iNOS, Cd80, and Cd86 and M1 cytokines, Tnf-a, Il-1b, Il-6, Il-12, and Il-23 in viperin KO M1 macrophages were significantly higher than those in WT M1 macrophages. Similar to these patterns, the expression levels of M2 macrophage markers, Cd163, Cd206, and Arg-1 and M2 cytokines, Il-10 and $T g f-b$ in viperin KO M2 macrophages were also increased when compared to those in WT M2 macrophages. The results indicated that viperin deficiency promotes the phenotypes of M1 and M2 macrophages, suggesting that viperin expression may play a key role as a regulator in macrophage polarization.

It is also known that interferon regulatory factors (IRFs) have a crucial role in the polarization of M1 and M2 macrophages (23-25). We examined gene expression of IRFs in WT and viperin KO M1 and M2 macrophages (Fig. 3D). The expression levels of Irf-1, Irf-5, and Irf-8 known as M1-related transcriptional factors and Irf-3 and Irf-4 known as M2-related transcriptional factors in WT M1 and M2 macrophages were similar to those of viperin KO macrophages. The results indicated that IRFs are upstream molecules of viperin in the IFN signaling pathway.

\section{Viperin deficiency enhances macrophage marker protein expression and cytokine secretion in the polarized M1 and M2 macrophages.}

To confirm the effect of viperin expression on macrophage polarization, we investigated M1 and M2 marker protein expression on cell surface and cytokine secretion using flow cytometry. The expression levels of M1 marker proteins, MHC class II and CD86 and an M2 marker protein, CD206 in viperin KO M1 and M2 macrophages were increased, when compared to those in WT macrophages (Fig. 4A). Furthermore, in cytometric bead array, the secretion levels of M1 cytokines, TNF- $\alpha$ and IL- 6 and an M2 cytokine, IL-10 in viperin KO M1 and M2 macrophages were higher than those in WT macrophages (Fig. 4B). The data indicated that viperin expression indeed regulates expression of macrophage markers and secretion of cytokines in the polarized M1 and M2 macrophages. 


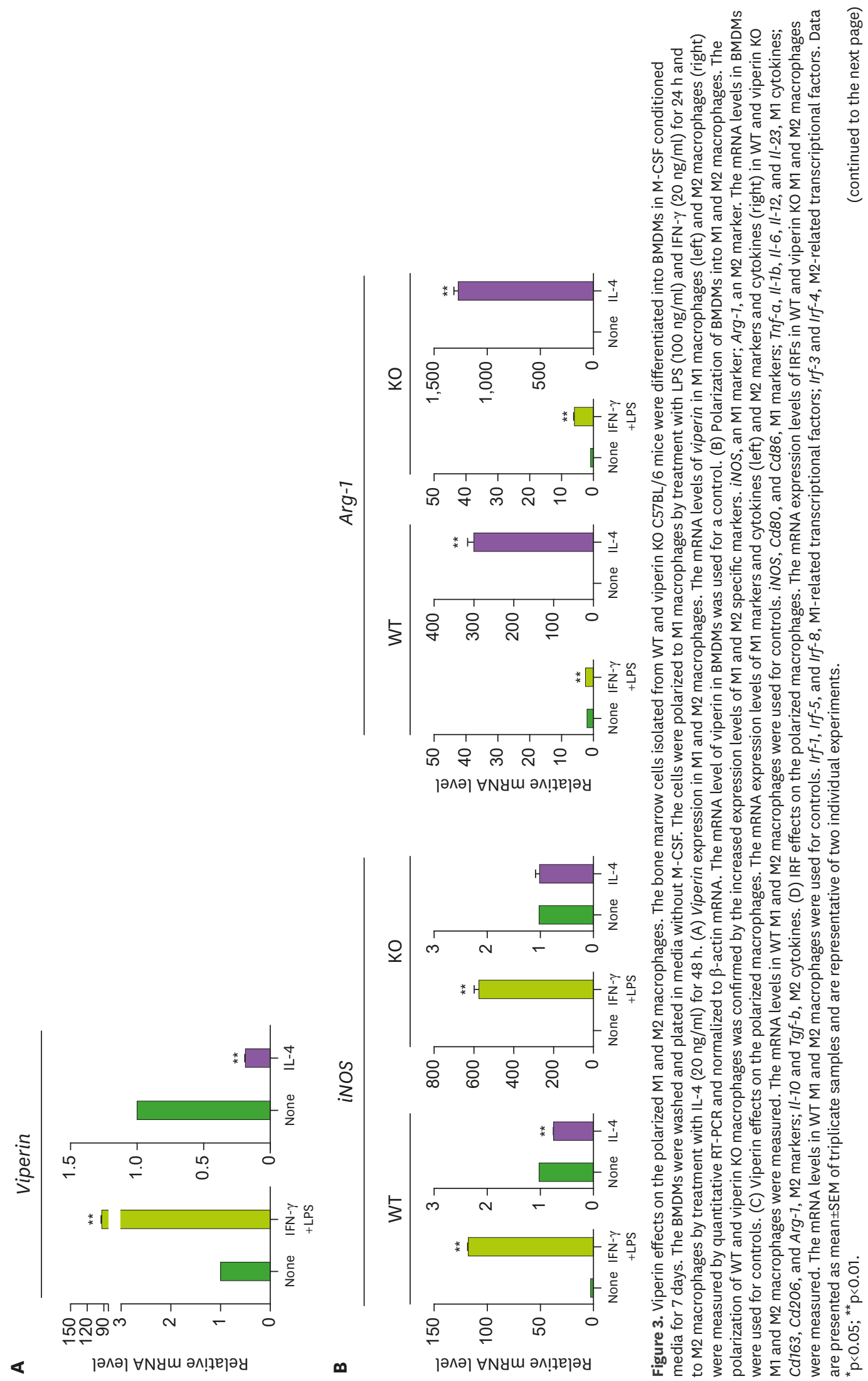



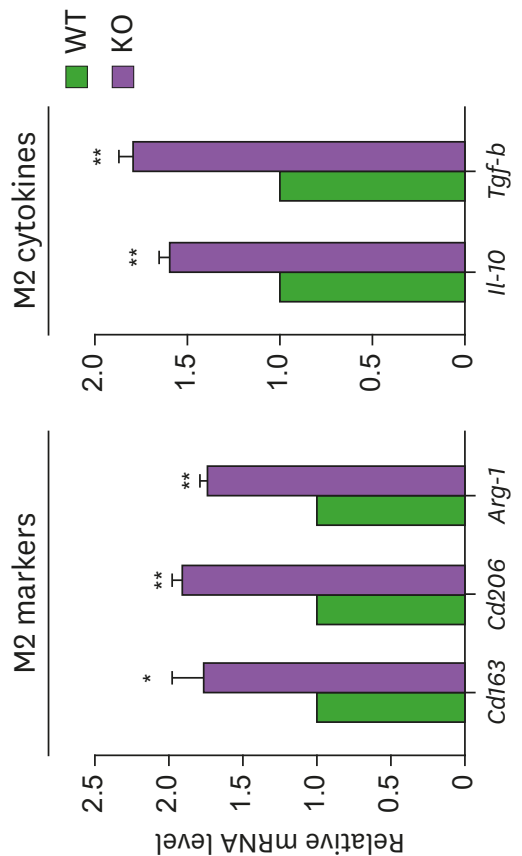

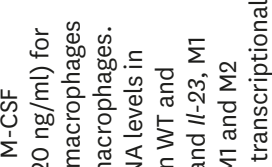

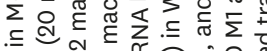

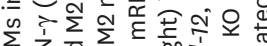

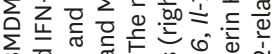

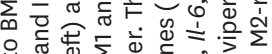

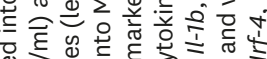

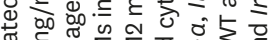

.

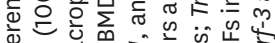

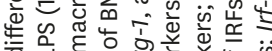

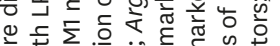

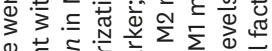

ษ

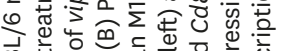

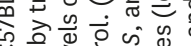

क ब

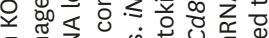

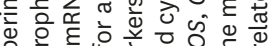

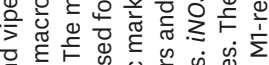

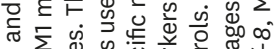

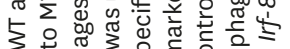

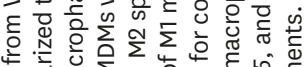

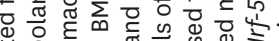

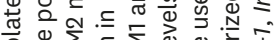

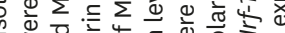

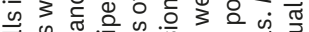

ত

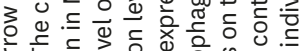

㲾

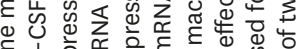

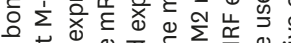

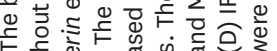

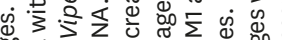

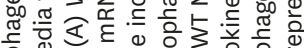

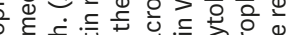

可.

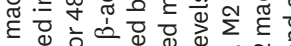

눈

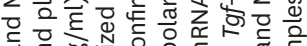

ब
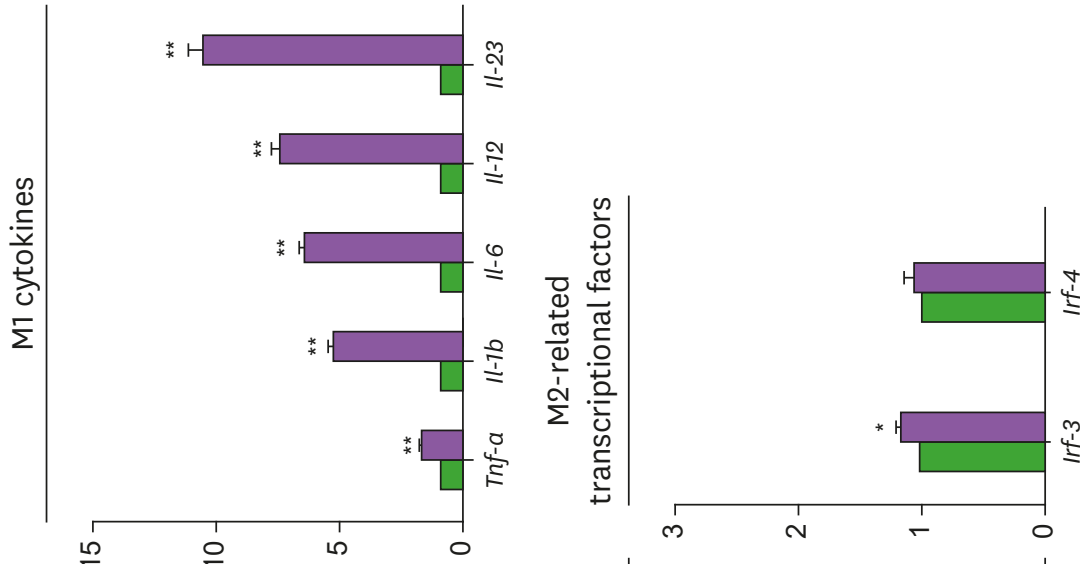

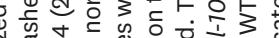

运 0

त

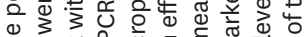

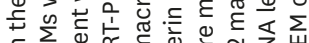

ธ

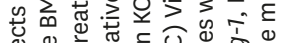

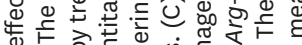

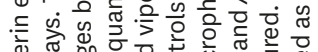

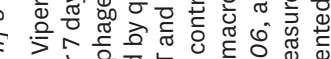

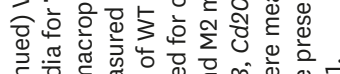

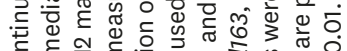

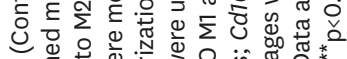

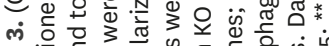

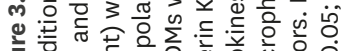
Mn

u

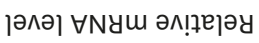

อ

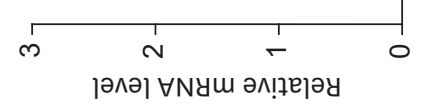
U. 
A

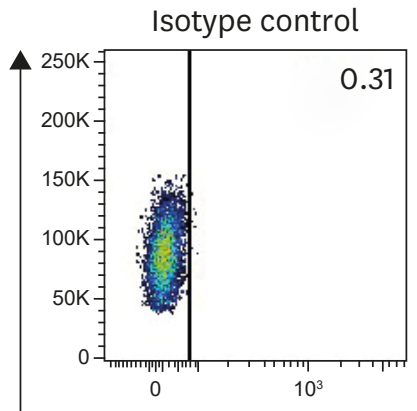

MHC II-FITC

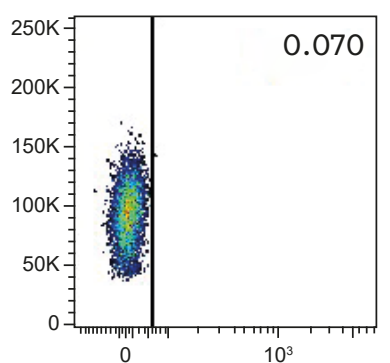

CD86-V450
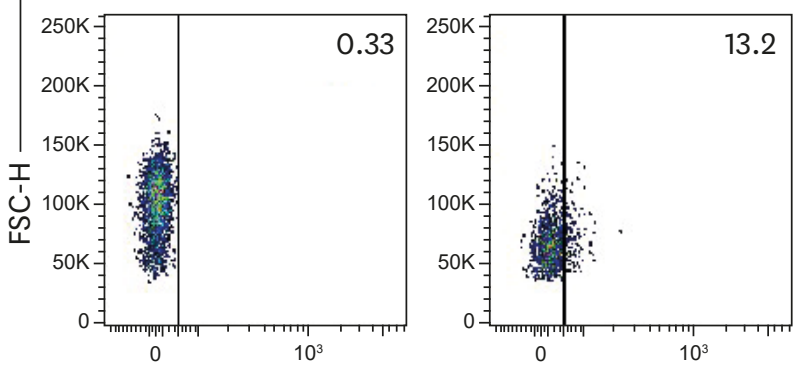

WT

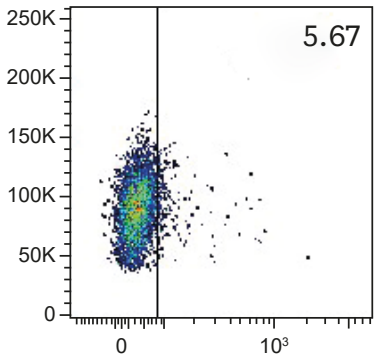

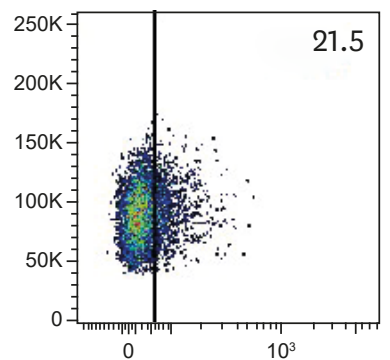

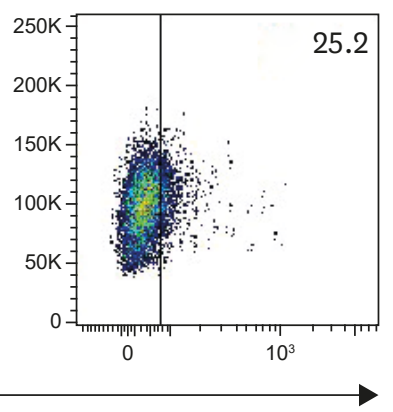

B

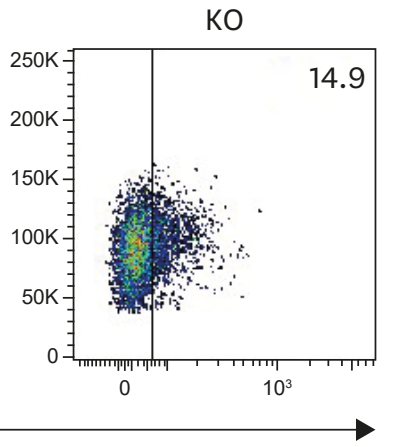

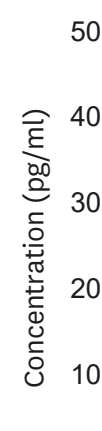

M1 cytokines

M2 cytokines
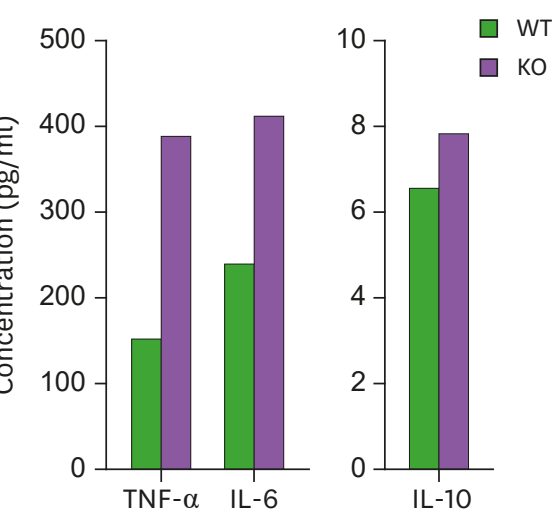

CD206-FITC

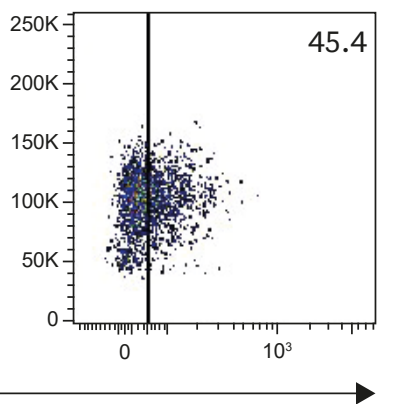

Figure 4. Viperin deficiency enhances expression of macrophage marker proteins and secretion of cytokines in the polarized macrophages. (A) The effect of viperin on expression of M1 and M2 marker proteins on cell surface. WT and viperin KO BMDMs were polarized into M1 and M2 macrophages as described above. The cells were stained with fluorescently-conjugated antibodies specific to MHC II, CD86, and CD206 and analyzed by flow cytometry. Left cytograms represent staining with an isotype-matched control antibody of irrelevant specificity. Right fraction on each cytogram indicates cell populations expressing M1 and M2 specific markers. MHC II and CD86, M1 markers; CD206, an M2 marker. (B) The effect of viperin on secretion of M1 and M2 cytokines. Cytometric bead array was performed. Open bars indicate cytokines secreted from WT M1 and M2 macrophages and closed bars represent cytokines secreted from viperin KO M1 and M2 macrophages. TNF- $\alpha$ and IL-6, M1 cytokines; IL-10, an M2 cytokine. Data are presented as concentration of cytokines secreted from M1 and M2 macrophages and are representative of 2 individual experiments.

FSC-H, forward scatter height.

\section{DISCUSSION}

Viperin is highly induced in primary human and mouse macrophages treated with IFN- $\gamma$ and in mouse macrophages of lymphoid organs acutely infected with lymphocytic choriomeningitis virus $(8,13,26)$. Our study showed that viperin is not only expressed in murine bone marrow cells, but also expressed in BMDMs differentiated with M-CSF in the absence of IFN treatment or virus infection. However, the basal expression of viperin did not affect the phenotypes, at least expression of macrophage markers and cytokines, between WT and viperin KO BMDMs. It suggested that: 1) the viperin expression level is not enough 
to alter the phenotypes of BMDMs, 2) viperin requires expression of other factors to affect the phenotypes of BMDMs, and 3) viperin expression might be involved in other functions including metabolic regulation rather than in immunological phenotypes of BMDMs. Furthermore, we demonstrated that viperin deficiency facilitates expression of macrophage markers and secretion of cytokines in M1 and M2 macrophages, implying a regulatory role of viperin in macrophage differentiation and polarization. The data suggested that viperin expression determines immunological functions of the completely differentiated macrophages, M1 and M2 macrophages which are activated with stimuli such as IFNs and virus-induced inflammatory cytokines in certain environment.

The main functions of macrophages are phagocytosis, endocytosis, secretion, and microbial killing (27). M1 and M2 macrophages have enhanced functional capacity compared with undifferentiated macrophages. Viperin effects on specific functions of M1 and M2 macrophages such as phagocytosis and endocytosis need to be further elucidated.

The role of viperin in M1 and M2 macrophages can be considered in macrophageassociated diseases such as asthma and atherosclerosis. When macrophages are exposed to local microenvironments, they are recruited to the sites and polarized into M1 or M2 phenotypes. Polarization of macrophages has been shown to have a crucial impact on asthma pathogenesis (28). Recent studies also showed that M2 macrophages play a central role in allergic asthma through their bidirectional interactions with immune cells and inflammatory mediators (29). In addition, it is well known that M1 macrophages are more likely to lead to an acute atherothrombotic vascular event (30), while M2 macrophages are associated with tissue repair and phagocytic capacities, enhancing plaque stability (31). A recent study showed that Irgm1 promotes murine M1 but not M2 macrophage polarization and its haplodeficiency renders ApoE KO mice resistant to atherosclerosis (32). Therefore, our data suggested that viperin regulation in macrophage polarization provides a potentially effective therapeutic strategy for macrophage-associated diseases.

Given the opposite roles of M1 and M2 macrophages in inflammatory responses and the effects of viperin on both of these polarized macrophages, viperin expression may act differently for the whole body in terms of protection or pathogenesis. Viperin expression may play a prophylactic role for inflammatory pathogenesis, including macrophage-associated diseases, by reducing the signature gene expression and cytokine production of M1 macrophages, although its expression can reduce the protective role of M1 macrophages in infections. On the other hand, viperin expression in M2 macrophages may function inversely.

In conclusion, we demonstrated that viperin participates in the polarization of macrophages. Viperin deficiency could increase the expression of M1 and M2 macrophage markers and cytokines. Our findings suggest that viperin may play a critical role in differentiation of macrophages and determination of phenotypes of M1 and M2 macrophages and provide clues to macrophage-related diseases.

\section{ACKNOWLEDGEMENTS}

We thank Drs. Kwang Hui Kim and Ki Taek Nam for assistance with cytometric bead array. This study was supported by grants from the National Research Foundation of Korea (NRF) funded by the Ministry of Science, ICT \& Future Planning (NRF-2016R1A2B4014630, NRF- 
2014R1A4A1008625, and 2013M3A9D5072550) and a faculty research grant from Yonsei University College of Medicine for 2013 and 2014 (6-2013-0063 and 6-2014-0063) to J.-Y. Seo, and the Brain Korea 21 PLUS Project for Medical Science, Yonsei University.

\section{REFERENCES}

1. Mosser DM, Edwards JP. Exploring the full spectrum of macrophage activation. Nat Rev Immunol 2008;8:958-969. PUBMED | CROSSREF

2. Gordon S, Martinez FO. Alternative activation of macrophages: mechanism and functions. Immunity 2010;32:593-604. PUBMED | CROSSREF

3. Liu YC, Zou XB, Chai YF, Yao YM. Macrophage polarization in inflammatory diseases. Int J Biol Sci 2014;10:520-529. PUBMED | CROSSREF

4. Wynn TA, Chawla A, Pollard JW. Macrophage biology in development, homeostasis and disease. Nature 2013;496:445-455. PUBMED | CROSSREF

5. Malyshev I, Malyshev Y. Current concept and update of the macrophage plasticity concept: intracellular mechanisms of reprogramming and $m 3$ macrophage "switch" phenotype. BioMed Res Int 2015;2015:341308. PUBMED | CROSSREF

6. Wang N, Liang H, Zen K. Molecular mechanisms that influence the macrophage M1-M2 polarization balance. Front Immunol 2014;5:614. PUBMED | CROSSREF

7. Porcheray F, Viaud S, Rimaniol AC, Léone C, Samah B, Dereuddre-Bosquet N, Dormont D, Gras G. Macrophage activation switching: an asset for the resolution of inflammation. Clin Exp Immunol 2005;142:481-489. PUBMED | CROSSREF

8. Chin KC, Cresswell P. Viperin (cig5), an IFN-inducible antiviral protein directly induced by human cytomegalovirus. Proc Natl Acad Sci U S A 2001;98:15125-15130. PUBMED | CROSSREF

9. Zhu H, Cong JP, Shenk T. Use of differential display analysis to assess the effect of human cytomegalovirus infection on the accumulation of cellular RNAs: induction of interferon-responsive RNAs. Proc Natl Acad Sci U S A 1997;94:13985-13990. PUBMED | CROSSREF

10. Seo JY, Yaneva R, Cresswell P. Viperin: a multifunctional, interferon-inducible protein that regulates virus replication. Cell Host Microbe 2011;10:534-539. PUBMED | CROSSREF

11. Jiang D, Guo H, Xu C, Chang J, Gu B, Wang L, Block TM, Guo JT. Identification of three interferoninducible cellular enzymes that inhibit the replication of hepatitis C virus. J Virol 2008;82:1665-1678. PUBMED | CROSSREF

12. Rivieccio MA, Suh HS, Zhao Y, Zhao ML, Chin KC, Lee SC, Brosnan CF. TLR3 ligation activates an antiviral response in human fetal astrocytes: a role for viperin/cig5. J Immunol 2006;177:4735-4741. PUBMED | CROSSREF

13. Wang X, Hinson ER, Cresswell P. The interferon-inducible protein viperin inhibits influenza virus release by perturbing lipid rafts. Cell Host Microbe 2007;2:96-105. PUBMED | CROSSREF

14. Zhang Y, Burke CW, Ryman KD, Klimstra WB. Identification and characterization of interferon-induced proteins that inhibit alphavirus replication. J Virol 2007;81:11246-11255. PUBMED | CROSSREF

15. Qiu LQ, Cresswell P, Chin KC. Viperin is required for optimal Th2 responses and T-cell receptor-mediated activation of NF- KB and AP-1. Blood 2009;113:3520-3529. PUBMED | CROSSREF

16. Saitoh T, Satoh T, Yamamoto N, Uematsu S, Takeuchi O, Kawai T, Akira S. Antiviral protein viperin promotes Toll-like receptor 7- and Toll-like receptor 9-mediated type I interferon production in plasmacytoid dendritic cells. Immunity 2011;34:352-363. PUBMED | CROSSREF 
17. Seo JY, Cresswell P. Viperin regulates cellular lipid metabolism during human cytomegalovirus infection. PLoS Pathog 2013;9:e1003497. PUBMED | CROSSREF

18. Seo JY, Yaneva R, Hinson ER, Cresswell P. Human cytomegalovirus directly induces the antiviral protein viperin to enhance infectivity. Science 2011;332:1093-1097. PUBMED | CROSSREF

19. Ryu SH, Na HY, Sohn M, Han SM, Choi W, In H, Hong S, Jeon H, Seo JY, Ahn J, et al. Reduced expression of granule proteins during extended survival of eosinophils in splenocyte culture with GM-CSF. Immunol Lett 2016;173:7-20. PUBMED | CROSSREF

20. Hamilton TA, Zhao C, Pavicic PG Jr, Datta S. Myeloid colony-stimulating factors as regulators of macrophage polarization. Front Immunol 2014;5:554.

PUBMED | CROSSREF

21. Hamilton JA, Achuthan A. Colony stimulating factors and myeloid cell biology in health and disease. Trends Immunol 2013;34:81-89.

PUBMED | CROSSREF

22. Hamilton JA. Colony-stimulating factors in inflammation and autoimmunity. Nat Rev Immunol 2008;8:533-544. PUBMED | CROSSREF

23. Martinez FO, Gordon S. The M1 and M2 paradigm of macrophage activation: time for reassessment. F1000Prime Rep 2014;6:13. PUBMED | CROSSREF

24. Günthner R, Anders HJ. Interferon-regulatory factors determine macrophage phenotype polarization. Mediators Inflamm 2013;2013:731023. PUBMED | CROSSREF

25. Chistiakov DA, Myasoedova VA, Revin VV, Orekhov AN, Bobryshev YV. The impact of interferonregulatory factors to macrophage differentiation and polarization into M1 and M2. Immunobiology 2018;223:101-111. PUBMED | CROSSREF

26. Hinson ER, Joshi NS, Chen JH, Rahner C, Jung YW, Wang X, Kaech SM, Cresswell P. Viperin is highly induced in neutrophils and macrophages during acute and chronic lymphocytic choriomeningitis virus infection. J Immunol 2010;184:5723-5731. PUBMED | CROSSREF

27. Mantovani A, Sica A, Sozzani S, Allavena P, Vecchi A, Locati M. The chemokine system in diverse forms of macrophage activation and polarization. Trends Immunol 2004;25:677-686. PUBMED | CROSSREF

28. Saradna A, Do DC, Kumar S, Fu QL, Gao P. Macrophage polarization and allergic asthma. Transl Res 2018;191:1-14. PUBMED | CROSSREF

29. Girodet PO, Nguyen D, Mancini JD, Hundal M, Zhou X, Israel E, Cernadas M. Alternative macrophage activation is increased in asthma. Am J Respir Cell Mol Biol 2016;55:467-475. PUBMED | CROSSREF

30. Kirbiš S, Breskvar UD, Šabovič M, Zupan I, Sinkovič A. Inflammation markers in patients with coronary artery disease--comparison of intracoronary and systemic levels. Wien Klin Wochenschr 2010;122 Suppl 2:31-34. PUBMED | CROSSREF

31. Habib A, Finn AV. The role of iron metabolism as a mediator of macrophage inflammation and lipid handling in atherosclerosis. Front Pharmacol 2014;5:195. PUBMED | CROSSREF

32. Fang S, Xu Y, Zhang Y, Tian J, Li J, Li Z, He Z, Chai R, Liu F, Zhang T, et al. Irgm1 promotes M1 but not M2 macrophage polarization in atherosclerosis pathogenesis and development. Atherosclerosis 2016;251:282-290. PUBMED | CROSSREF 